\title{
Systemic Analysis on Laparoscope-Assisted Gastrectomy for Patients with Gastric Cancer
}

\author{
Jun-Hong Hu ${ }^{\circledR}$, Hong-Na Tang ${ }^{\star}$, Yong-Ping Ma , Chen-Yu Wang, Kun-Hou Yao, \\ Jun-Jie Zhang, Xue-Qun Ren*
}

\begin{abstract}
Background: Laparoscope-assisted gastrectomy in treating patients with gastric cancers developed with a background of highly invasive traditional surgery and is being increasingly performed in the Asian Pacific area. This study systemically investigated the technique and clinical results for comparison with traditional radical subtotal gastrectomy for gastric cancers. Methods: Clinical studies evaluating the effectiveness and side effects of laparoscope-assisted gastrectomy in treating patients with gastric cancers were identified using a predefined search strategy. Summary rates of effectiveness and side effects of laparoscope-assisted gastrectomy were calculated. Results: Thirteen clinical studies which including 1,412 patients with gastric cancer treated by laparoscope-assisted gastrectomy were considered eligible for inclusion. Systemic analysis showed that, for all patients, the pooled resection rate was $100 \%$. Major adverse effects were anastomotic stenosis, abdominal abscess, abdominal bleeding, postoperative ileus. Treatment related death occurred in $0.71 \%(10 / 1412)$. Conclusion: This systemic analysis suggests that laparoscope-assisted gastrectomy in treating patients with gastric cancers is associated with good curative rate and acceptable complications.
\end{abstract}

Keywords: Laparoscope-assisted gastrectomy - gastric cancer - cure rate - complications

Asian Pac J Cancer Prev, 16 (5), 2027-2029

\section{Introduction}

Gastric cancer (GC) is one of the most common cancer, and according to GLOBOCAN 2008, 989 thousand new cases (including 640 thousand males and 349 thousand females) and 738 thousand GC deaths (including 464 thousand males and 273 thousand females) occurred worldwide (Ferlay et al., 2010). The disease is also very common in China. It ranked the third most common cancer in Chinese population and estimated new cases of and deaths from GC were about 400 and 300 thousand respectively per year (Yang et al., 2005; Yang, 2006). The disease is also very common in China. It ranked the third most common cancer in Chinese population and estimated new cases of and deaths from GC were about 400 and 300 thousand respectively per year. In the treatment setting, it has been reported that laparoscopic technique for surgically treating patients with gastric cancer is a minimally invasive approach, and has shown oncologic and long-term survival equivalency to the open technique . However, many Chinese patients with gastric cancer were diagnosed at a late stage of disease, the same as compared with patients in other part of the world. That is estimated that $68 \%$ of patients diagnosed with gastric carcinoma are in advanced-stage, with poor treatment results (Wei et al., 2013; Xu et al., 2013; Liu et al., 2014). On this background, an increasing number of surgeons have become concerned about laparoscopic surgery for advanced gastric cancer (AGC). It would seem more important to study laparoscopic techniques for AGC. D2 lymph node dissection showed the benefits for fit patients with early- and intermediate-stage disease, for the reason that $\mathrm{D} 2$ dissection was possible to remove more positive nodes than D1 dissection. But D2 dissection was also thought to be a more appropriate treatment for patients with advanced disease, $\mathrm{i}$

n centers that could demonstrate low operative mortality. The Japanese gastric cancer treatment guidelines have adopted D2 lymph node dissection as the standard operation. However, debate on oncological adequacy and postoperative outcomes makes the use of laparoscopyassisted gastrectomy (LAG) with D2 lymph node dissection for AGC still controversial, it remains questionable on account of the technical difficulty of D2 lymph node dissection. Therefore, we hypothesized before this systemic analysis that there should be value of LAG for treating patients with gastric cancer.

\section{Materials and Methods}

Search strategy

We searched PUBMED, by using the following search

Department of General Surgery, Huaihe Hospital of Henan University, Kaifeng, Henan, China ${ }^{\star}$ Equal contributors ${ }^{*}$ For correspondence: xue_qun769@126.com 
term: (laparoscope assisted gastrectomy) and (gastric cancer). All clinical studies evaluating the impact of laparoscope-assisted gastrectomy on the treatment result, survival and side effects for patients with gastric cancer published in English prior to November 1st, of 2014 were identified. If samples of two studies overlap, only the latest one was included. Additional articles were obtained from references within the articles identified by the electronic search. We did not consider meeting abstracts or unpublished reports.

\section{Inclusion and exclusion criteria}

We reviewed abstracts of all citations and retrieved studies. The following criteria were used to include published studies: (1) clinical studies, combined with paclitaxel or pirarubicin; (2) The study was performed in accordance with the Helsinki Declaration (1964, amended in 1975 and 1983) of the World Medical Association. Eligibility criteria included histologically or cytologically verified with gastric cancer, the presence of a disease located in the stomach, a performance status (WHO) 2 , age $<18$ years. Studies were excluded if one of the following existed: (1) duplicate data; (2) no sufficient data were reported.

\section{Data collection and analysis}

Selection of trials and data extraction: The titles and abstracts of publications identified according to the above search strategy were assessed independently for inclusion by two authors; the full text was selected for further assessment if the abstract suggests relevance. Disagreement was resolved by discussion. Data was extracted by independent authors. The following recorded data were extracted: author, publication data, and country of the first or corresponding author, the number of patients.

\section{Results}

There were 22 papers relevant to the search words by the end of November 1st, of 2014. Via steps of screening the title and reading the abstract, 3 studies were identified (Huang YLet al., 2014; Huang Z et al., 2014; Yang et al., 2014) when LAG was used to treat gastric cancer. These studies had been carried out in China. The following outcomes were presented in at least all studies and extracted for combined analysis: safety and efficacy of treatment. When LAG was used to treat gastric cancer, 3 studies included in this study are presented and the mortality rate of Huang $\mathrm{Z}$ et al.was $1.7 \%$, of Yang et al.was $1.5 \%$, and of Huang YL et al.was $0.51 \%$. Totally, pooled resection rate was $100 \%$. Major adverse effects were anastomotic stenosis, abdominal abscess, abdominal bleeding, postoperative ileus. Treatment related death occurred in $0.71 \%(10 / 1412)$.

\section{Discussion}

LAG combined with regional lymph node dissection is an alternative surgical treatment for patients with gastric cancer and such operation increases significantly in these ten years in the world (Lee et al., 2005; Kinoshita et al.,
2011; Ahn et al., 2012). It is notable that LAG is not widely conducted due to the fact that only experienced surgeons could perform it (Cho et al., 2009; Kawamura et al., 2009). However, compared with conventional open total gastrectomy, LAG is an advanced procedure and offers the advantages of less invasiveness and comparable resection rate on the condition that surgeons are adroit at performing LAG (Eom et al., 2012). Another concern is the treatment effect between LAG and open operation.

Huang $\mathrm{Z}$ et al., evaluated the clinical feasibility of laparoscope-assisted D2 radical total gastrectomy for gastric cancer. They enrolled 216 gastric cancer patients who underwent D2 radical total gastrectomy, including 118 laparoscopic and 98 open procedures, compared intraoperative and postoperative outcomes. Compared with open surgery group, LAG group had longer operation time [ $(253.9 \pm 26.1) \mathrm{min}$ vs. $(206.2 \pm 23.9) \mathrm{min}$, $p<0.01]$, less intraoperative blood loss[ $(138.4 \pm 34.0)$ $\mathrm{ml}$ vs. $(266.3 \pm 58.7) \mathrm{ml}, p<0.01]$, shorter postoperative hospital stay [ $(10.3 \pm 1.4) \mathrm{d}$ vs. $(13.9 \pm 2.0) \mathrm{d}, p<0.01]$, and lower morbidity of postoperative complication[16.1\% (19/118) vs. $29.6 \%$ (29/98), $p<0.05$ ] (Huang Z et al., 2014). No significant differences in the number of lymph node removed [ $(26.2 \pm 6.2$ vs. 27.6 \pm 5.6$)]$, postoperative morbidity of complication [1.7\% (2/118) vs. $2.0 \%(2 / 98)]$, and 2-year survival rate $(99.0 \%$ vs. $98.6 \%)$ between the two groups (all $p>0.05$ ) was found (Huang $\mathrm{Z}$ et al., 2014). In conclusion, they suggested that compared to open surgery, LAG is safe, feasible with the advantage of rapid postoperative recovery (Huang $\mathrm{Z}$ et al., 2014). In this field, Huang YL et al conducted a meta-analysis to evaluate the value of $L A G$ versus $O G$ with regard to safety and efficacy for treatment of ADG (Huang YL et al., 2014). They identified studies that compared LAG and OG with D2 lymph node dissection for treatment of ADG, and selected eligible 11 studies with totally 1904 patients (982 in LAG and 922 in OG). Pooled risk ratios (RR) and weighted mean difference (WMD) with $95 \%$ confidence intervals (CI) were appropriately derived from random-effects models or fixed-effects models (Huang YL et al., 2014). Compared with OG, LAG was associated with less blood loss (WMD $=-144.47 ; p<0.05)$, shorter time of first flatus time $(\mathrm{WMD}=-0.91 ; p<0.05)$ and postoperative hospital stay (WMD $=-3.27 ; p<0.05)$, and lower morbidity $(\mathrm{RR}=0.70 ; p<0.05)$, but longer operation time $(\mathrm{WMD}=41.78 ; p<0.05)$. No significant differences were noted in terms of harvested lymph nodes (WMD= $1.85 ; p=0.09)$, pathological N stage $\left(\chi^{2} 3.97 ; p=0.26\right)$, tumor size $(\mathrm{WMD}=-0.05 ; p=0.81)$, mortality $(\mathrm{RR} 0.82$; $p=0.76)$, cancer recurrence rate (RR $0.77 ; p=0.18)$ and 3 -year overall survival rate (RR 1.09; $p=0.18$ ) (Huang YL et al., 2014). Compared with OG, LAG with D2 lymph node dissection for ADG had the advantages of minimal invasion, faster recovery, and fewer complications, and it could achieve the same degree of radicality, harvested lymph nodes, short-term and long-term prognosis as OG, but the operation time was slightly longer (Huang YL et al., 2014), especially in patients with obesity. Yang $\mathrm{H}$ et al.To investigate the influence of obesity on laparoscopy-assisted radical gastrectomy, they collected clinical data of 214 patients with gastric cancer, who 
underwent laparoscopy-assisted radical gastrectomy retrospectively (Yang et al., 2014). In their study, patients were divided into two groups, consisting of obese and non-obese. In the obese group, the BMI was $\geq 25.0 \mathrm{~kg} /$ $\mathrm{m}^{2}(\mathrm{n}=66)$, and in the non-obese group was $<25.0 \mathrm{~kg} /$ $\mathrm{m}^{2}(\mathrm{n}=148)$. Based on their results, operative time was longer in obese group than that in non-obese group [ (271.5 \pm 51.2$) \mathrm{min}$ vs. $(252.1 \pm 53.6) \mathrm{min}, p<0.05$ ] (Yang et al., 2014). The number of retrieved lymph nodes in obese group was less than that in non-obese group $(26.2 \pm 10.3$ vs. $30.3 \pm 12.4, p<0.05)$. No significant differences were observed in terms of blood loss, blood transfusion rate, conversion to laparotomy and time to first flatus between these two groups (all $p>0.05$ ) (Yang et al., 2014). There were no significant differences between the two groups with respect to postoperative complications rate $(25.8 \%$ vs. $20.9 \%, p>0.05)$ and perioperative mortality $(1.5 \%$ vs. $0.7 \%, p>0.05)$. However, minor surgery-related complication rate was higher in obese group $(16.7 \%$ vs. $6.8 \%, p<0.05)$, mainly presented as delayed gastric emptying (Yang et al., 2014). There was no difference in perioperative mortality between the two groups ( $1.5 \%$ vs. $0.7 \%, p>0.05$ ) (Yang et al., 2014). In conclusion, Yang $\mathrm{H}$ et al.suggested that obesity prolongs the duration of laparoscopy-assisted radical gastrectomy, and increases the risk of minor surgery-related complications, and it has no influence on the surgical safety (Yang et al., 2014).

In our current study, we used a predefined searching strategy to collect clinical studies evaluating the effectiveness and side effects of LAG in treating patients with gastric cancers. Summary rates of effectiveness and side effects of laparoscope-assisted gastrectomy were calculated.Our results suggested that for 1412 patients with gastric cancer treated by LAG, pooled resection rate was $100 \%$. Major adverse effects were anastomotic stenosis, abdominal abscess, abdominal bleeding, postoperative ileus. Treatment related death occurred in $0.71 \%(10 / 1412)$. Thus we concluded that LAG is associated with good curative rate and acceptable complications for treating patients with gastric cancer.

\section{Acknowledgements}

The research was supported by Ministry of Health-The Standardization study of minimally invasive surgery for cancer (No: W2013R67).

\section{References}

Ahn SH, Lee JH, Park DJ, et al (2012). Comparative study of clinical outcomes between laparoscopy-assisted proximal gastrectomy (LAPG) and laparoscopy-assisted total gastrectomy (LATG) for proximal gastric cancer. Gastric Cancer. 16, 282-9.

Cho HJ, Kim W, Hur H, et al (2009). Laparoscopy-assisted completion total gastrectomy for gastric cancer in remnant stomach: report of 2 cases. Surg Laparosc Endosc Percutan Tech, 19, 57-60.

Eom BW, Kim YW, Lee SE, et al (2012). Survival and surgical outcomes after laparoscopy-assisted total gastrectomy for gastric cancer: case-control study. Surg Endosc, 26, 3273-81.

Ferlay J, Shin HR, Bray F, et al (2010). Estimates of worldwide burden of cancer in 2008: GLOBOCAN 2008. Int J Cancer, 127, 2893-917.

Kawamura H, Yokota R, Homma S, et al (2009). Comparison of invasiveness between laparoscopy-assisted total gastrectomy and open total gastrectomy. World J Surg, 33, 2389-95.

Huang YL, Lin HG , Yang JW , et al (2014). Laparoscopy-assisted versus open gastrectomy with D2 lymph node dissection for advanced gastric cancer: a meta-analysis. Int J Clin Exp Med, 7, 1490-9.

Huang Z, Li G, Xu Y, et al (2014). Comparison of laparoscopeassisted D2 radical total gastrectomy and open gastrectomy for gastric cancer. Zhonghua Wei Chang Wai Ke Za Zhi, 25, 781-4.

Kinoshita T, Shibasaki H, Oshiro T, et al (2011). Comparison of laparoscopy-assisted and total laparoscopic Billroth-I gastrectomy for gastric cancer: a report of short-term outcomes. Surg Endosc, 25, 1395-401.

Lee JH, Han HS, Lee JH (2005). A prospective randomized study comparing open vs laparoscopy-assisted distal gastrectomy in early gastric cancer: early results. Surg Endosc, 19, 168-73.

Liu J, Huang XE, Tian GY, et al (2013). Phase II study on safety and efficacy of Yadanzi? (Javanica oil emulsion injection) combined with chemotherapy for patients with gastric cancer. Asian Pac J Cancer Prev, 14, 2009-12.

Wei GL, Huang XE, Huo JG, et al (2013). Phase II study on pemetrexed-based chemotherapy in treating patients with metastatic gastric cancer not responding to prior palliative chemotherapy. Asian Pac J Cancer Prev, 14, 2703-6.

$\mathrm{Xu} \mathrm{X,} \mathrm{Wang} \mathrm{L,} \mathrm{Xu} \mathrm{HQ,} \mathrm{et} \mathrm{al} \mathrm{(2013).} \mathrm{Clinical} \mathrm{comparison}$ between paclitaxel liposome (Lipusu?) and paclitaxel for treatment of patients with metastatic gastric cancer. Asian Pac J Cancer Prev, 14, 2591-4.

Yang H, Xing J, Cui M, et al (2014). Efficacy evaluation of laparoscopy-assisted radical gastrectomy in obese patients with gastric cancer. Zhonghua Wei Chang Wai Ke Za Zhi, 17, 776-80.

Yang L, Parkin DM, Ferlay J, et al (2005). Estimates of cancer incidence in China for 2000 and projections for 2005. Cancer Epidemiol Biomarkers Prev, 14, 243-50.

Yang L (2006). Incidence and mortality of gastric cancer in China. World J Gastroenterol, 12, 17-20. 